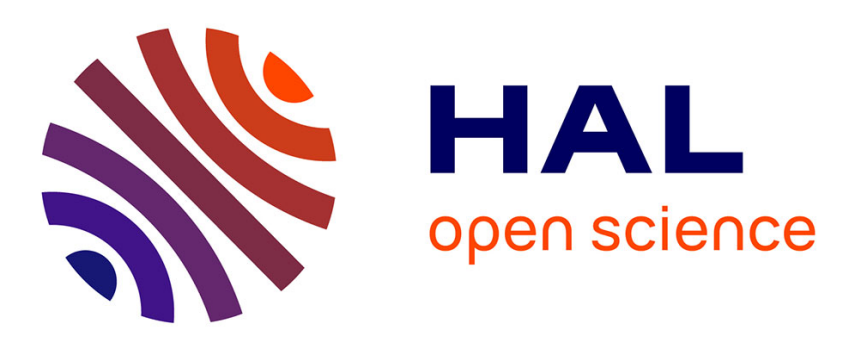

\title{
Blind DFE based on NLMS algorithm with generalized normalized gradient descent regularization
}

\author{
Abderrazek Abdaoui, Christophe Laot
}

\section{To cite this version:}

Abderrazek Abdaoui, Christophe Laot. Blind DFE based on NLMS algorithm with generalized normalized gradient descent regularization. Ocean's 2009, Oct 2009, Biloxi, United States. hal-01893757

\section{HAL Id: hal-01893757 \\ https://hal.science/hal-01893757}

Submitted on 11 Jun 2021

HAL is a multi-disciplinary open access archive for the deposit and dissemination of scientific research documents, whether they are published or not. The documents may come from teaching and research institutions in France or abroad, or from public or private research centers.
L'archive ouverte pluridisciplinaire HAL, est destinée au dépôt et à la diffusion de documents scientifiques de niveau recherche, publiés ou non, émanant des établissements d'enseignement et de recherche français ou étrangers, des laboratoires publics ou privés. 


\title{
Blind DFE based on NLMS Algorithm with Generalized Normalized Gradient Descent Regularization
}

\author{
Abderrazak Abdaoui and Christophe Laot \\ Institut TELECOM; TELECOM Bretagne; UMR CNRS 3192, Lab-STICC \\ Université européenne de Bretagne \\ Technopole Brest-Iroise - CS 83818 - 29238 Brest Cedex 3, France \\ Abderrazek.abdaoui@telecom-bretagne.eu,Christophe.Laot@telecom-bretagne.eu
}

\begin{abstract}
This paper presents robust unsupervised decision feedback equalizer (DFE) for acoustic underwater communications. The proposed equalizer consists of the cascade of four devices whose main components are recursive $(\mathcal{R})$ and transverse $(\mathcal{T})$ filters. The feature of the given equalizer is the ability to deal with severe quickly time varying channels by allowing the adjustment of both, its structure and its adaptation according to a mean square error (MSE) criterion. In the existing solution, the recursive and transverse filters are updated by decision directed least-mean-square (LMS) algorithms. However, the weakness of the LMS like algorithms against the time varying environments pushes us to improve the adaptation by the use of other robust solutions. In this paper, we propose the employ of normalized LMS algorithms with self step-size regularization based on complexvalued generalized normalized gradient descent (GNGD) method instead of simple LMS algorithms. Compared to the existent unsupervised DFE, the proposed solution gives the best performance in channel tracking despite the irregularities and the nonstationarity of the environment. Performance analysis are given in terms of the MSE for both synthetic and realistic channels obtained from underwater acoustic recorded signals.
\end{abstract}

\section{INTRODUCTION}

Present underwater communications systems involve the transmission of information using either acoustic or optical techniques. Among the solutions proposed for acoustic communications, Labat, Macchi and Laot have introduced an unsupervised decision feedback equalizer (DFE) based on least mean square (LMS) algorithms [5]. This equalizer has been implemented in underwater acoustic modem called Trident which has given high performance and has been tested on horizontal configurations at low depth [6][7]. The proposed equalizer is able to adjust, in a reversible way, both its structure and its adaptation according to the mean-square error (MSE) measure. The unsupervised DFE includes gain control component $\mathcal{G C}$, recursive filter $\mathcal{R}$, and transversal filter $\mathcal{T}$. The unsupervised starting period begins with $\mathcal{R}$ before $\mathcal{T}$. Once the MSE is below a fixed threshold, the equalizer is switched into a classical DFE-decision directed simply by placing $\mathcal{R}$ after $\mathcal{T}$.

The performance of the unsupervised DFE equalizer depends on the adaptation of the filter coefficients in the blocks $\mathcal{R}$ and $\mathcal{T}$. Hence the weakness of the LMS against the time varying environments pushes us to look for other LMS and NLMS filters with auto-regularized step-sizes.

Usually, in standard LMS algorithm, the optimum value of the step-size parameter $\mu$ cannot be known a priori due to

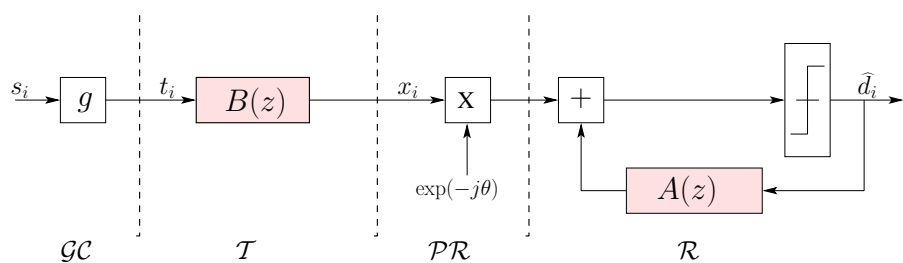

Fig. 1. Structure of the DFE based equalizer in the tracking period.

the channel knowlegeness. To overcome this weakness, some variable step-size (VSS) LMS algorithms have been developed in last few years. Some of these algorithms are Mathew's and Benveniste's algorithms [8] which are based on gradient descent technique to adjust the step-size parameter $\mu$. There exist other variants of Mathew's and Beneviste algorithms. One of them uses multiple $\mu$ to update the different filter weights resulting a multiple step-size VLMS [9][10]. The main disadvantage of the algorithms based on the minimization of the squared error upon the step-size $\mu$ is their sensitivity to the time correlation between input signal samples and the value of the additional step-size related to the gradient adaptation of the step-size.

Furthermore, conventional NLMS algorithm can be modified to improve its diverging nature by introducing a small constant value in NLMS update equation. This value can be made adaptive using the generalized normalized gradient descent (GNGD) NLMS algorithm [11]. Another solution, based on GNGD approach and gives a simplification for the GNGD, is called $\varepsilon$-NLMS. To make the regularization of $\varepsilon$ adaptive, the authors in [11], introduce a normalized gradient into the update scheme of $\varepsilon$. It is demonstrated that the proposed method is computationally efficient and outperforms the conventional NLMS in terms of convergence speed and misadjustment error for speech input signals. However, in their proposed form, the GNGD-NLMS and the $\varepsilon$-NLMS [12] algorithms operate on real-valued signals which limits their applications.

In this paper, we propose the use of normalized LMS algorithm with self step-size regularization based on complexvalued GNGD method. To this end, we reformulate the analytical derivations of the GNGD-NLMS and $\varepsilon$-NLMS for complex-valued signals. Throughout this work, the GNGDNLMS is employed in $\mathcal{R}$ and $\mathcal{T}$ blocks during tracking 
mode only. Compared with the existent unsupervised DFE, the proposed solution gives the best performances in tracking mode despite the irregularities and the non-stationarity of the channel.

The remainder of the paper is as follows. Section II presents the details of the proposed complex-valued GNGD-NLMS algorithm. The $\varepsilon$-NLMS, is presented in section III. Numerical results, related to the performances of the blind DFE with Monte-Carlo simulations and experimental sea trials are detailed and analyzed in Section IV. We conclude the paper in Section V.

\section{GNGD NLMS COMPLEX-VALUED ALGORITHM}

In this section, we detail the proposed GNGD-NLMS complex-valued algorithm. The GNGD-NLMS was first proposed by Mandic [11] in order to introduce adaptivity in the variable step-size parameter. In time varying environments, NLMS equalizer requires an adaptivity in the step-size in order to track the system variabilities. This algorithm is basically defined as in standard LMS algorithm; it uses the same weightupdate mechanism, as can be seen in the following equations

$$
\begin{aligned}
e_{i} & =d_{i}-\mathbf{w}_{i}^{H} \mathbf{u}_{i} \\
\mathbf{w}_{i+1} & =\mathbf{w}_{i}+\frac{\widetilde{\mu}}{\left\|\mathbf{u}_{i}\right\|^{2}+\varepsilon} e_{i}^{*} \mathbf{u}_{i}
\end{aligned}
$$

The adaptation of the instantaneous $\mu=\frac{\widetilde{\mu}}{\left\|\mathbf{u}_{i}\right\|^{2}+\varepsilon}$ is applied for the regularization parameter $\varepsilon$ by minimising the criterion $J(i)=\frac{1}{2}\left|e_{i}\right|^{2}, \varepsilon$ is then adapted as

$$
\varepsilon(i)=\varepsilon(i-1)-\rho \nabla_{\varepsilon(i-1)} J(i)
$$

where $\rho$ is some small constant that adjusts the adaptation of $\varepsilon$. In the following, we detail the derivation of $\nabla_{\varepsilon(i-1)} J(i)$ for the general complex-valued signals.

$$
\nabla_{\varepsilon} J(i)=-\frac{1}{2}\left[e_{i}\left(\frac{\partial \mathbf{w}_{i}}{\partial \varepsilon(i-1)}\right)^{T} \mathbf{u}_{i}^{*}+e_{i}^{*}\left(\frac{\partial \mathbf{w}_{i}}{\partial \varepsilon(i-1)}\right)^{H} \mathbf{u}_{i}\right]
$$

where $\frac{\partial \mathbf{w}_{i}}{\partial \varepsilon(i-1)}$ is easily computed as

$$
\frac{\partial \mathbf{w}_{i}}{\partial \varepsilon(i-1)}=-\frac{\tilde{\mu}}{\left(\left\|\mathbf{u}_{i-1}\right\|^{2}+\varepsilon(i-1)\right)^{2}} e_{i-1}^{*} \mathbf{u}_{i-1}
$$

Finally, we substitute (4) and its complex conjugate in (3) we have

$$
\begin{aligned}
\nabla_{\varepsilon} J(i)= & \frac{1}{2}\left[e_{i} \frac{\widetilde{\mu} e_{i-1}^{*} \mathbf{u}_{i-1}^{T}}{\left(\left\|\mathbf{u}_{i-1}\right\|^{2}+\varepsilon(i-1)\right)^{2}} \mathbf{u}_{i}^{*}+\right. \\
& \left.e_{i}^{*} \frac{\widetilde{\mu} e_{i-1} \mathbf{u}_{i-1}^{H}}{\left(\left\|\mathbf{u}_{i-1}\right\|^{2}+\varepsilon(i-1)\right)^{2}} \mathbf{u}_{i}\right] \\
= & \widetilde{\mu} \Re e\left[\frac{e_{i}^{*} e_{i-1} \mathbf{u}_{i-1}^{H} \mathbf{u}_{i}}{\left(\left\|\mathbf{u}_{i-1}\right\|^{2}+\varepsilon(i-1)\right)^{2}}\right]
\end{aligned}
$$

Hence, the complex-valued GNGD-NLMS algorithm is given as

$$
\begin{aligned}
& \text { 1. } \varepsilon(i)=\varepsilon(i-1)-\rho \widetilde{\mu} \Re e\left[\frac{e_{i}^{*} e_{i-1} \mathbf{u}_{i-1}^{H} \mathbf{u}_{i}}{\left(\left\|\mathbf{u}_{i-1}\right\|^{2}+\varepsilon(i-1)\right)^{2}}\right] \\
& \text { 2. } \quad e_{i}=d_{i}-\mathbf{w}_{i}^{H} \mathbf{u}_{i} \\
& \text { 3. } \quad \mathbf{w}_{i+1}=\mathbf{w}_{i}+\frac{\widetilde{\mu}}{\left\|\mathbf{u}_{i}\right\|^{2}+\varepsilon(i)} e_{i}^{*} \mathbf{u}_{i}
\end{aligned}
$$

\section{RobUSt REgULARIZATION $\varepsilon$-NLMS}

The $\varepsilon$-NLMS, dynamically updates the regularization parameter which is fixed in the basic NLMS. To make the regularization parameter robust, Choi and Song [13] introduce the normalized gradient into the update scheme for the regularization parameter. They show that the proposed method is computationally efficient compared to the GNGD-NLMS algorithm.

In $\varepsilon$-NLMS, the adaptation of $\varepsilon$ against the channel variabilities is performed by minimizing $J(i)=\frac{1}{2}\left|e_{i}\right|^{2}$. To achieve this goal, the regularization parameter $\varepsilon$ is updated by a gradient descent manner. Thus, $\varepsilon$ is recursively adapted as

$$
\varepsilon(i)=\varepsilon(i-1)-\rho \frac{\nabla_{\varepsilon} J(i)}{\left\|\nabla_{\varepsilon} J(i)\right\|}
$$

It is noticed that $\nabla_{\varepsilon} J(i)$ is normalized by its norm. Unsing the same derivation of $\nabla_{\varepsilon(i-1)} J(i)$ as in (5) we obtain

$$
\frac{\nabla_{\varepsilon} J(i)}{\left\|\nabla_{\varepsilon} J(i)\right\|}=\operatorname{sign}\left(\Re e\left[\frac{e_{i}^{*} e_{i-1} \mathbf{u}_{i-1}^{H} \mathbf{u}_{i}}{\left(\left\|\mathbf{u}_{i-1}\right\|^{2}+\varepsilon(i-1)\right)^{2}}\right]\right)
$$

where $\operatorname{sign}(x)$ is the sign function. Finally, the complex $\varepsilon$ NLMS algorithm can be summarized as follows

$$
\begin{aligned}
& \text { 1. } \varepsilon^{\prime}(i)=\varepsilon(i-1)-\rho \operatorname{sign}\left(\Re e\left\{\left(e_{i}^{*} e_{i-1} \mathbf{u}_{i-1}^{H} \mathbf{u}_{i}\right)\right\}\right) \\
& \text { 2. } \quad \varepsilon(i)= \begin{cases}\varepsilon^{\prime}(i) ; \text { for } \varepsilon^{\prime}(i) \geq \varepsilon_{\min } \\
\varepsilon_{\min } \quad ; \text { for } \varepsilon^{\prime}(i)<\varepsilon_{\min }\end{cases} \\
& \text { 3. } e_{i}=d_{i}-\mathbf{w}_{i}^{H} \mathbf{u}_{i} \\
& \text { 4. } \quad \mathbf{w}_{i+1}=\mathbf{w}_{i}+\frac{\tilde{\mu}}{\left\|\mathbf{u}_{i}\right\|^{2}+\varepsilon(i)} e_{i}^{*} \mathbf{u}_{i}
\end{aligned}
$$

\section{Numerical Results}

\section{A. Performances on synthetic signals}

The data symbols $d_{n}$ are QPSK symbols with unity variance at a rate $1 / T=5 k$ bauds. The multipath channel is a three paths each with Bello fading model [14]. The channel impulse response is then given by

$$
h(t)=a_{1}(t) \delta(t)+a_{2}(t) \delta(t-2 T)+a_{3}(t) \delta(t-4 T)
$$

where $T$ is the delay between two successive paths and $a_{i}(t)$ are the complex path gains assumed to be uncorrelated with one another. The fading properties of the channel coefficients, will depend on the Doppler spread parameter $\sigma_{f}^{2}$. We denote 
by $\varepsilon_{\mathcal{R}}$ and $\varepsilon_{\mathcal{T}}$ the regularization parameters of the filters in the recursive $\mathcal{R}$ and transverse $\mathcal{T}$ blocks respectively. The parameters $\rho_{\mathcal{R}}$ and $\rho_{\mathcal{T}}$ related to the adaptation of $\varepsilon_{\mathcal{R}}$ and $\varepsilon_{\mathcal{T}}$ respectively and defined in (2), are chosen to achieve the best MSE. The initial values of $\varepsilon_{\mathcal{R}}$ and $\varepsilon_{\mathcal{T}}$ for the GNGD-NLMS are chosen to be 0 as in [11]. However for $\varepsilon$-NLMS, $\varepsilon_{\min }$ related to the filters in $\mathcal{R}$ and $\mathcal{T}$ are chosen to optimize the MSE and the stability of the whole unsupervised DFE. In the following, for each scenario, we give the instantaneous channel variations, (as the real part of each channel coefficient) and the MSE performances evaluated by a mean of 200 realizations each with $2.10^{4}$ symbols.

Throughout the numerical results, the instantaneous stepsize values in the NLMS, $\varepsilon$-NLMS and GNGD-NLMS algorithms depend on the filter length, which is $(\mathrm{N}=5)$ for $\mathcal{T}$ and $(\mathrm{L}=20)$ for $\mathcal{R}$. Specifically, the step-size values in LMS are chosen to be $\mu_{l m s} \simeq \frac{\widetilde{\mu}_{\mathcal{T}}}{L \sigma_{d}^{2}}$ for transverse filter and $\mu_{l m s} \simeq \frac{\widetilde{\mu}_{\mathcal{R}}}{N \sigma_{d}^{2}}$ for recursive filter, where $\widetilde{\mu}_{\mathcal{T}}$ and $\widetilde{\mu}_{\mathcal{R}}$ are the step-size parameters in NLMS, $\varepsilon$-NLMS and GNGD-NLMS algorithms emplyed to update the transverse and the recursive filters respectively.

In Figs. 2 and 3, under low average signal to noise ratio (SNR) of $10 \mathrm{~dB}$ and channel variations defined by Doppler spread vector $[0,1.5,0.5] \mathrm{Hz}$ (for slow channel variations) and $[0,2.5,1.5] \mathrm{Hz}$ (for fast channel variations), the unsupervised DFE based on NLMS algorithms have the same behavior and they guarantee the minimum MSE. However, the DFE with LMS algorithm have slightly poorer performance considering its fixed step-size. In each figure, the green curve (MSE $\mathrm{M}_{\text {limit }}$ ) represents the MSE performances obtained by optimum equalizer where

$$
\operatorname{MSE}_{\text {limit }}(t)=\frac{\sigma_{d}^{2} \sigma_{n}^{2}}{\sigma_{n}^{2}+\sum_{i=1}^{K}\left|a_{i}(t)\right|^{2} \sigma_{d}^{2}}
$$

$K$ is the number of paths and $\sigma_{d}^{2}$ and $\sigma_{n}^{2}$ represent the energy of the transmitted data symbols and the noise respectively.

From Fig. 2 to 3, we have evaluated the performances of the DFE with respect to the dynamics of the channel. We can notice that, in order to maintain the same performances from slow to fast channel, we have to, slightly, increase the stepsize for each algorithm. In fact, for fast time varying channels, blind DFE requires LMS algorithms with fast responses, and this can be ensured by the increase of the step-size values. However, the choices of the step-size values have to be controlled by the stability condition of each algorithm.

In Figs. 4 and 5, we evaluate the performances of the unsupervised DFE under high average SNR of $15 \mathrm{~dB}$ and channel variations fixed by Doppler spread vector $[0,1.5,0.5]$ $\mathrm{Hz}$ (for slow channel variations) and $[0,2.5,1.5] \mathrm{Hz}$ (for fast channel variations). For $\mathrm{SNR}=15 \mathrm{~dB}$, the unsupervised $\mathrm{DFE}$ based on NLMS algorithms still the best compared to the LMS based equalizer. The DFE with LMS algorithm have slightly poorer performance considering its fixed step-size. From Fig. 4 to 5, we can notice also that more dynamic channels require the use of step-sizes with improved values.
In fact, for fast time varying channels, blind DFE equalization requires fast response in LMS algorithms, and this can be ensured by the increase of the step-size values. These choices have to be controlled by the stability conditions imposed by each algorithm.

Mainly, we can notice that the proposed algorithms do not allow excellent channel tracking, even the GNGD is slightly better.

\section{B. Performances on signals from sea trials}

Experimental sea trials were carried out the 10th October 2006 in the site "anse de Camaret" near Brest, France, by GESMA/DGA. The aim of these sea trials was to transmit SONAR images in real-time from an autonomous underwater vehicle (AUV) to a ship [15], [16]. The transmitter and the receiver were placed on the AUV and the ship, respectively. A QPSK transmission with carrier frequency of $35 \mathrm{kHz}$ was used during the trials.

In order to emphasize on the performance of the proposed adaptive algorithms, we show the tracking behavior of the equalizer output on a trial of duration 10 seconds. For this trial, the distance between the transmitter and the receiver was about $1200 \mathrm{~m}$, the depth around was $20 \mathrm{~m}$, the bit rate was 9.85 kbps. The receiver has 4 sensors and includes demodulation, timing recovery and blind adaptive DFE.

$\mathrm{L}=20$ coefficients in the transversal filters, $\mathrm{N}=15$ coefficients in the recursive part. In GNGD-NLMS: $\rho=0.15, \widetilde{\mu}_{\mathcal{T}}=0.06$ and $\widetilde{\mu}_{\mathcal{R}}=0.045$ and for LMS: $\mu_{\mathcal{T}}=0.003$ and $\mu_{\mathcal{R}}=0.003$. In Fig. 6, GNGD performs slightly better than LMS. In Fig. 7 , we have enlarged the plots.

\section{CONCLUSION}

In this paper, unsupervised DFE based on LMS adaptation is improved by the use of more adaptive NLMS like algorithms such as GNGD-NLMS. We have shown that, under fast channel variations, the GNGD NLMS algorithm increases the tracking capacity and let a minimum MSE in the unsupervised DFE. For the unsupervised DFE, GNGD-NLMS algorithm ensure a minimum MSE and good stability against the channel variations.

The performances are given assuming Bello fading channels with slow and fast variations. In addition, the unsupervised DFE with GNGD-NLMS is tested on underwater acoustic recorded signals which give a great confirmation with the results obtained by synthetic channels. In future work, we can look for more realistic channel model like sparse channels and the slight adaptation in the algorithms by the use of sparse criterion. Otherwise, considering the results obtained by LMS, NLMS and GNGD algorithms, we have tested iterative techniques (iterative block processing) and we notice that a significant gain of some $\mathrm{dB}$ on the MSE can be achieved and thus the MSE is slightly close to $\mathrm{MSE}_{\text {limit }}$ curve.

In overall, in order to rich optimal performances, we have to seek for more robust algorithms for the unsupervised DFE with time varying and non stationary channels. 


\section{ACKNOWLEDGMENT}

This work has been supported by GESMA — DGA, France

\section{REFERENCES}

[1] H.-C. Shin, A. H. Sayed, and W.-J. Song, "Variable step-size NLMS and affine projection algorithms," IEEE Signal Processing Letters, vol. 11, no. 2, pp. 132-135, Feb. 2004.

[2] H. Rey, L. R. Vega, S. Tressens, and J. Benesty, "Optimum variable explicit regularized affine projection algorithm," in Proc. IEEE International Conference on Acoustics, Speech and Signal Processing ICASSP 2006, vol. 3, 14-19 May 2006, pp. III-III.

[3] S. Haykin, Adaptive Filter Theory, 4th ed. New Jersey, USA: Prentice Hall, September 2001.

[4] J. Benesty, H. Rey, L. R. Vega, and S. Tressens, "A Nonparametric VSS NLMS Algorithm," IEEE Signal Processing Letters, vol. 13, no. 10, pp. 581-584, Oct. 2006.

[5] J. Labat, O. Macchi, and C. Laot, "Adaptive decision feedback equalization: Can you skip the training period," IEEE Transactions on Communications, vol. 46, no. 7, pp. 921-930, Jul 1998.

[6] J. Trubuil, G. Lapierre, J. Labat, N. Beuzeulin, A. Goalic, and C. Laot, "Improved AUV autonomy provided by an underwater acoustic link," in Proc. International Society of Offshore and Polar Engineers ISOPE 2006, San Francisco USA, 28 May-2 June, 2006.

[7] G. Lapierre, J. Labat, and J. Trubuil, "Iterative equalization for underwater acoustic channel ? potentiality for the TRIDENT system," in in Proc. OCEANS'03, San Diego, USA, 2003, pp. 1547-1553.

[8] A. Benveniste, M. Metivier, and P. Priouret, Adaptive Algorithms and Stochastic Approximation. New York: Springer-verlag, 1990.

[9] V. J. Mathews and Z. Xie, "A stochastic gradient adaptive filter with gradient adaptive step size," IEEE Transactions on Signal Processing, vol. 41, no. 6, pp. 2075-2087, June 1993.

[10] B. Farhang-Boroujeny, "Variable-step-size LMS algorithm: new developments and experiments," IEE Proceedings -Vision, Image and Signal Processing, vol. 141, no. 5, pp. 311-317, Oct. 1994.

[11] D. P. Mandic, "A general nomalised grandient descent algorithm," IEEE Signal Processing Letters, vol. 11, no. 2, pp. 115-118, Feb. 2004.

[12] D. P. Mandic, D. Obradovic, and A. Kuh, "A robust general normalized gradient descent algorithm," in Proc. IEEE 13th Workshop on Statistical Signal Processing,, Bordeau France, 17-20 Jul, 2005.

[13] Y.-S. Choi, H.-C. Shin, and W.-J. Song, "Robust regularization for Normalized LMS Algorithm," IEEE Transactions on Circuits and SystemsPart II: Express Briefs, vol. 53, no. 8, pp. 627-631, Aug 2006.

[14] P. Bello, "Characterization of randomly time-variant linear channels," IEEE Transactions on Communications, vol. CS, no. 11, pp. 360-393, Dec. 1963.

[15] G. Lapierre, N. Beuzelin, J. Labat, J. Trubuil, A. Goalic, S. Saoudi, G. Ayela, P. Coince, and S. Coatelan, "1995-2005: ten years of active research on underwater acoustic communications in Brest," in Proc. of OCEANS'05, Brest, France, 2005, pp. 425- 430.

[16] J. Trubuil, G. Lapierre, J. Labat, N. Beuzelin, A. Goalic, and C. Laot, "Improved AUV autonomy provided by an underwater acoustic link," in Proc. of ISOPE'06, San Francisco, USA, 2006.
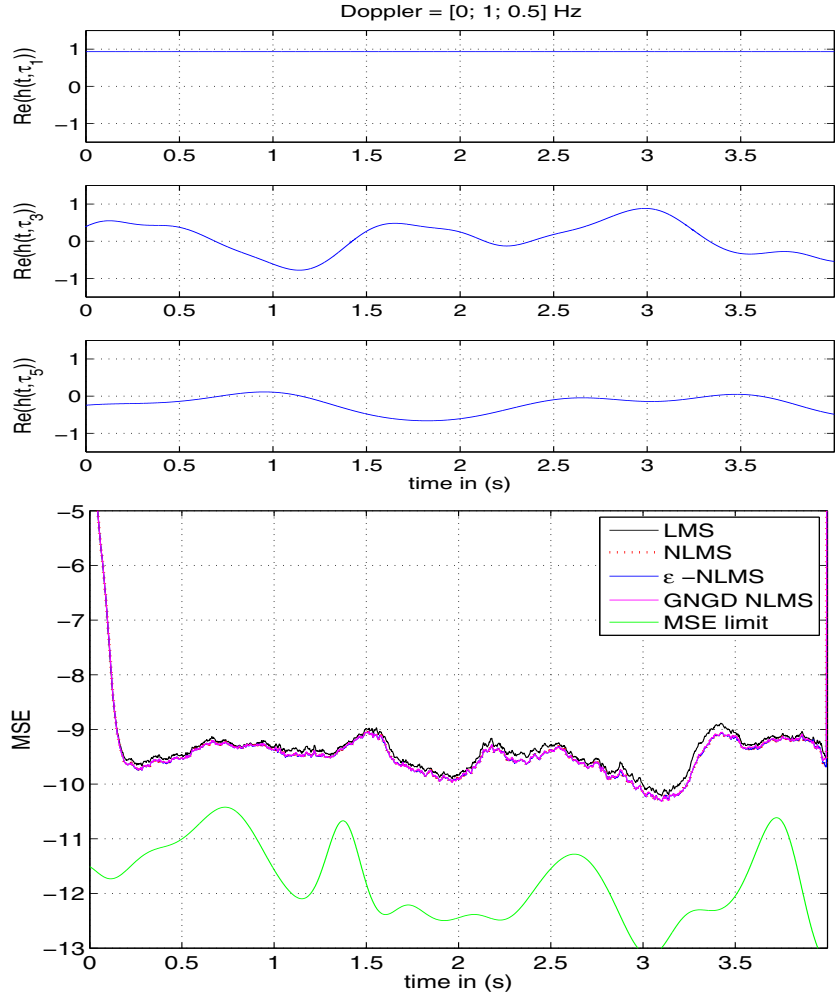

Fig. 2. MSE, for the DFE based on LMS $\left(\mu_{\mathcal{T}}=0.005, \mu_{\mathcal{R}}=0.0268\right)$, NLMS $\left(\widetilde{\mu}=0.11, \varepsilon=10^{-5}\right), \varepsilon$-NLMS $\left(\widetilde{\mu}=0.11 \varepsilon_{\text {min }}=10^{-5}\right.$, $\left.\rho=10^{-4}\right)$ and GNGD $\left(\tilde{\mu}=0.11, \varepsilon_{0}=10^{-6}, \rho=5\right)$, SNR=10 dB.
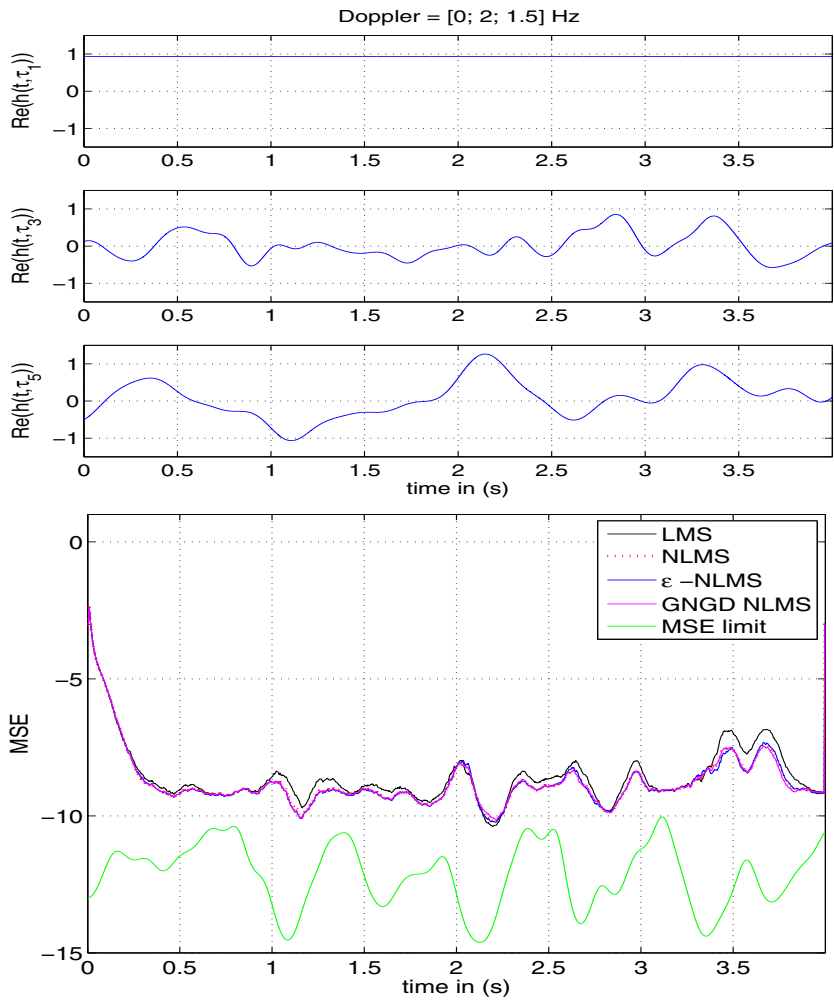

Fig. 3. MSE, for the DFE based on LMS $\left(\mu_{\mathcal{T}}=0.00625, \mu_{\mathcal{R}}=0.025\right)$, NLMS $\left(\widetilde{\mu}=0.125, \varepsilon=10^{-2}\right), \varepsilon$-NLMS $\left(\widetilde{\mu}=0.125, \varepsilon_{\min }=10^{-6}\right.$, $\left.\rho=10^{-4}\right)$ and GNGD $\left(\widetilde{\mu}=0.125, \varepsilon_{0} \simeq 0, \rho=1\right), \mathrm{SNR}=10 \mathrm{~dB}$. 

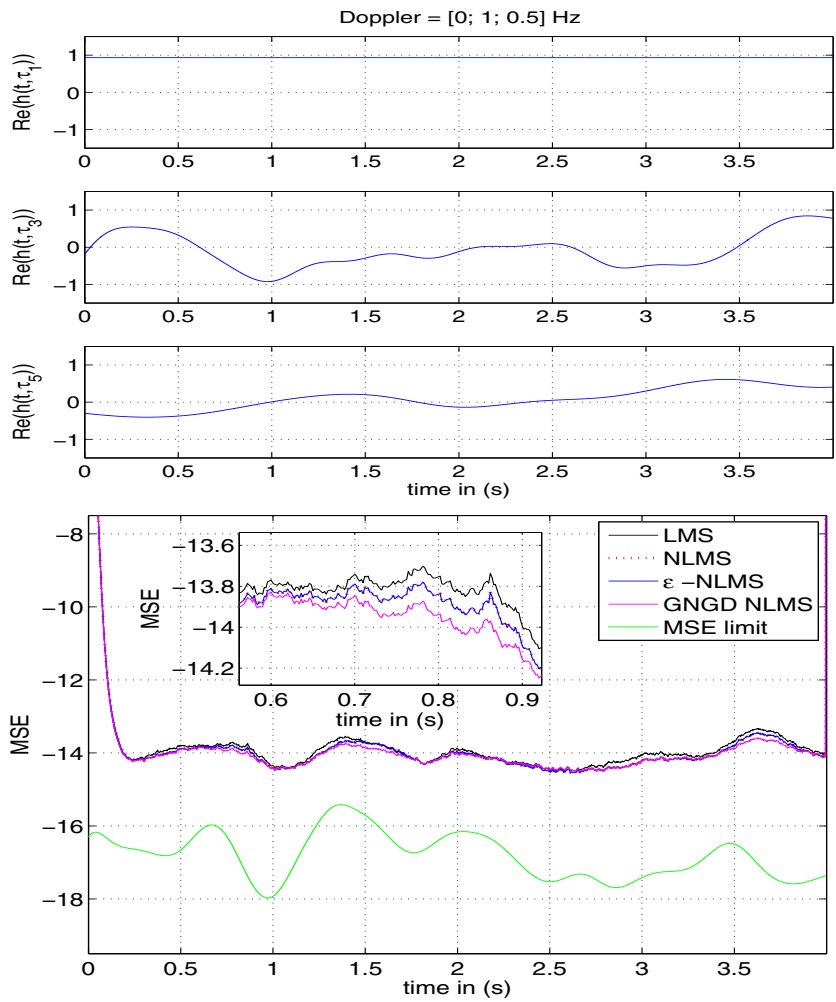

Fig. 4. MSE, for the DFE based on LMS $\left(\mu_{\mathcal{T}}=0.00363, \mu_{\mathcal{R}}=0.0154\right)$, $\left(\widetilde{\mu}_{\mathcal{T}}=0.08, \widetilde{\mu}_{\mathcal{R}}=0.11\right)$, NLMS $\left(\varepsilon=10^{-5}\right), \varepsilon$-NLMS $\left(\varepsilon_{\min }=10^{-5}\right.$, $\left.\rho=10^{-4}\right)$ and GNGD $\left(\varepsilon_{0} \simeq 0, \rho=1\right)$.
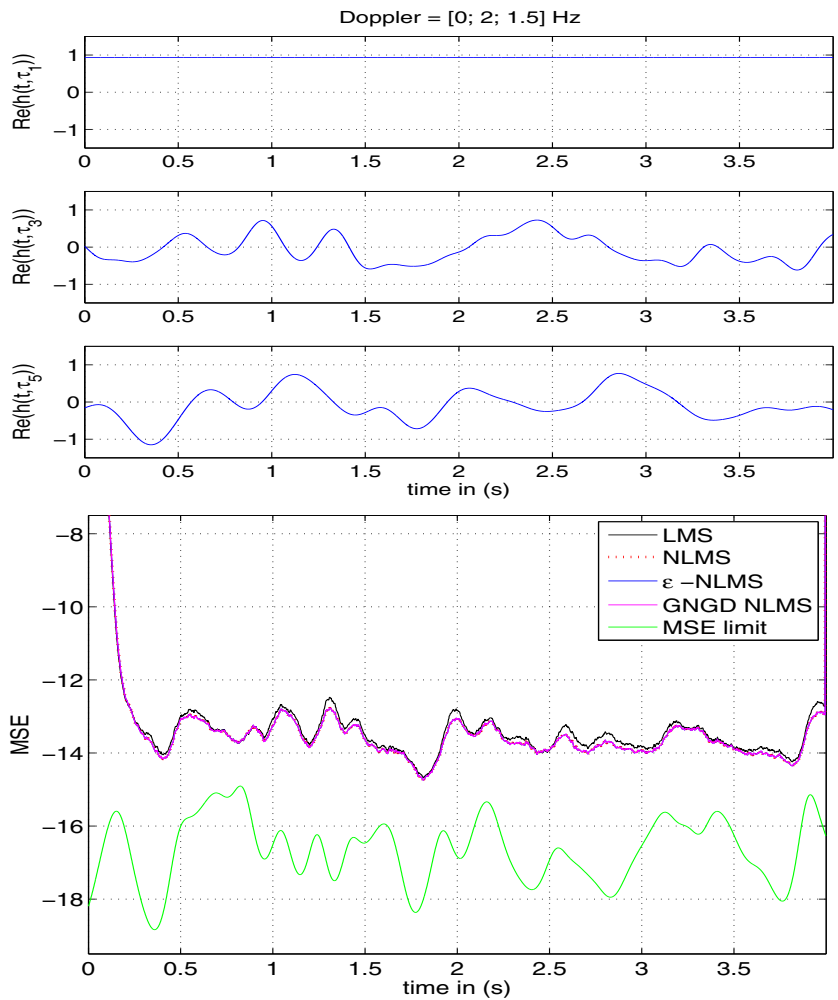

Fig. 5. MSE, for the DFE based on LMS $\left(\mu_{\mathcal{T}}=0.00625, \mu_{\mathcal{R}}=0.025\right)$, $\left(\widetilde{\mu}_{\mathcal{T}}=0.125, \widetilde{\mu}_{\mathcal{R}}=0.125\right)$, NLMS $\left(\varepsilon=10^{-5}\right), \varepsilon$-NLMS $\left(\varepsilon_{\min }=10^{-5}\right.$, $\left.\rho=10^{-4}\right)$ and $\operatorname{GNGD}\left(\varepsilon_{0} \simeq 0, \rho=1\right)$.

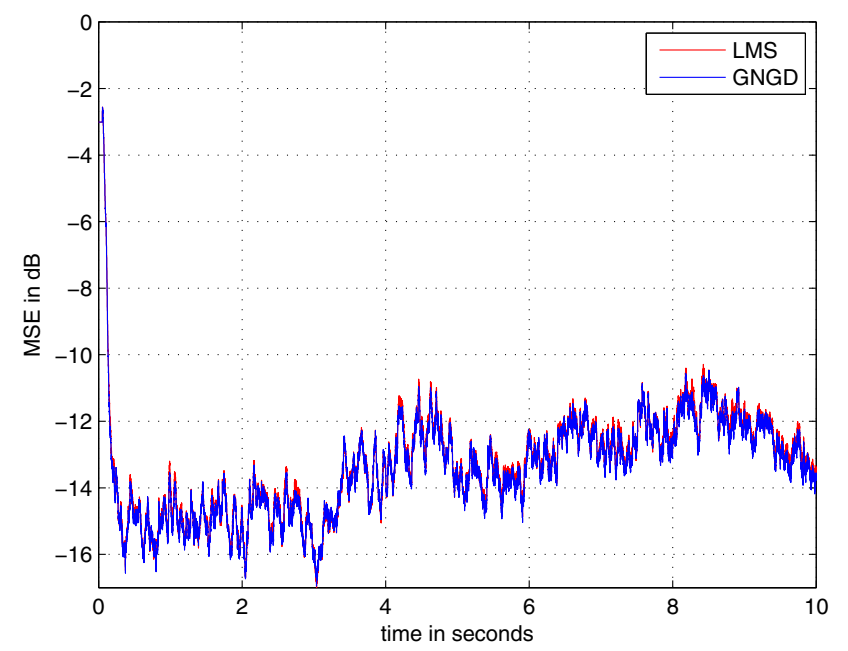

Fig. 6. MSE performance in experimental sea trials

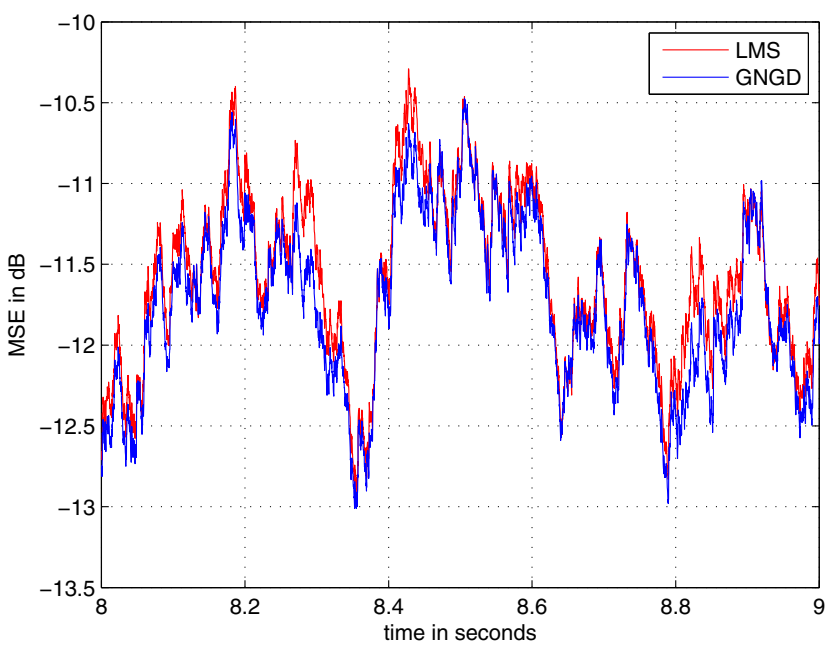

Fig. 7. MSE performance in experimental sea trials 